\title{
Effect of statins on platelet function in patients with hyperlipidemia
}

\author{
Joanna Sikora ${ }^{1}$, Barbara Kostka², Iwona Marczyk³, Urszula Krajewska², Maciej Chałubiński², \\ Marlena Broncel ${ }^{3}$
}

\begin{abstract}
1Department of Pharmaceutical Chemistry and Drug Analyses, Medical University of Lodz, Poland

2Department of Pharmaceutical Biochemistry, Medical University of Lodz, Poland ${ }^{3}$ Department of Internal Diseases and Clinical Pharmacology, Medical University of Lodz, Poland
\end{abstract}

Submitted: 19 July 2011

Accepted: 26 September 2011

Arch Med Sci 2013; 9, 4: 622-628

DOI: 10.5114 /aoms.2013.36905

Copyright (c) 2013 Termedia \& Banach

\author{
Corresponding author: \\ Dr Joanna Sikora \\ Department of \\ Pharmaceutical Chemistry \\ and Drug Analyses \\ Medical University of Lodz \\ 1 Muszynskiego St \\ 90-151 Lodz, Poland \\ Phone: + 48426779295 \\ E-mail: joanna.sikora@ \\ umed.lodz.pl
}

\begin{abstract}
Introduction: It is generally assumed that cholesterol reduction by statins is the predominant therapeutic result underlying their beneficial effects in cardiovascular disease. However, the action of statins may be partially independent of their effects on plasma cholesterol levels, as they combine lipid lowering with positive effects on hemorheological conditions and endothelial function. We evaluated the impact of statin treatment on platelet adhesion to fibrinogen (spontaneous and ADP-activated), along with ADP, collagen or ristocetin-induced aggregation in type II hyperlipidemic patients.

Material and methods: The study group included 70 persons: 50 patients affected by type II hyperlipidemia without concomitant diseases and 20 healthy volunteers. The effects of 8 -week statin treatment (atorvastatin $10 \mathrm{mg} / \mathrm{day}$, simvastatin $20 \mathrm{mg} /$ day, or pravastatin $20 \mathrm{mg} /$ day) on platelet activation were evaluated.

Results: Regardless of the type of statin, a significant decrease in ADP-induced platelet aggregation was observed: for atorvastatin $50.6 \pm 12.8 \%$ vs. $41.1 \pm 15.8 \%$ ( $p<0.05$ ), for simvastatin $57.2 \pm 18.0 \%$ vs. $44.7 \pm 22.1 \%(p=0.05)$, and for pravastatin $55.8 \pm 19.5 \%$ vs. $38.8 \pm 23.3 \%(p<0.05)$. There was no significant effect of statins on collagen or ristocetin-induced platelet aggregation and adhesion. Conclusions: Therapy with statins beneficially modifies ADP-induced platelet aggregation in patients with hyperlipidemia and does not affect spontaneous or ADP-induced platelet adhesion to fibrinogen and platelet aggregation induced by collagen or ristocetin.
\end{abstract}

Key words: hyperlipidemia, platelet adhesion, platelet aggregation, statins.

\section{Introduction}

Platelets play a pivotal role in the process of thrombogenesis, and take part in atherogenesis and the progression of atherosclerotic lesions [1]. Activated platelets adhering to impaired endothelium, subendothelium or blood and vessel wall cells promote thrombus formation and the release of vasoactive mediators inducing vasoconstriction and remodeling of the vessel wall [2]. Normal vascular endothelium prevents adhesion and aggregation of platelets by way of nitric oxide (NO) production, from constitutive nitric oxide synthase (cNOS) action on L-arginine, and the release of 
prostacyclin [2, 3]. Oxidant species and high levels of low-density lipoprotein cholesterol (LDL-C), particularly its oxidized forms (oxLDL-C), were shown to decrease cNOS expression and enhance thrombus formation [4]. The LDL-C was found to promote platelet aggregation, when induced by agonists or by themselves at concentrations of more than $1 \mathrm{mg}$ protein/ml. Independently, at high concentrations, LDL-C enhanced the exposure to fibrinogen binding sites on platelets and the secretion of dense granules [5].

Hypercholesterolemia may be associated with an increase in platelet reactivity. This abnormality is linked to the rise of the cholesterol/phospholipid ratio in platelets. Other potential mechanisms include an increase in thromboxane $A_{2}$ biosynthesis, platelet $\alpha_{2}$-adrenergic receptor density, and platelet cytosolic calcium [6].

Some researchers have failed to detect native LDL-induced changes of platelet aggregability or have reported inhibition of ADP and thrombininduced aggregation [7].

Recent trials have suggested that the beneficial effects of statins may be partially independent of their effects on plasma cholesterol levels, as they combine lipid lowering with positive effects on hemorheological conditions, endothelial function as well as effector and regulatory immune mechanisms $[8,9]$.

The aim of our study was to evaluate the impact of atorvastatin (10 mg/day), simvastatin (20 mg/ day) and pravastatin (20 mg/day) on platelet adhesion to fibrinogen (with or without ADP), along with $A D P$, collagen or ristocetin-induced aggregation in type II hyperlipidemic subjects.

\section{Material and methods}

\section{Reagents}

Platelet agonists used for the study were freshly prepared from stock solutions: adenosine 5'-diphosphate - ADP (Sigma-Aldrich, Munich, Germany), collagen (Chrono-Log, Havertown, Pennsylvania, USA), ristocetin (Boehringer Mannheim, Wien, Austria), and arachidonic acid (Sigma-Aldrich, Munich, Germany). ADP and ristocetin stock solutions were stored in small $(20 \mu \mathrm{l})$ frozen $\left(-70^{\circ} \mathrm{C}\right)$ portions. Collagen stock solution was stored at $4^{\circ} \mathrm{C}$. Human fibrinogen was obtained from Kabi (Uppsala, Sweden).

Atorvastatin (Sortis) was purchased from ParkeDavis/Pfizer, simvastatin (Zocor) was from Merck Sharp \& Dohme and pravastatin (Lipostat) was from Bristol-Myers Squibb.

\section{Patients}

The study group included 70 persons: $50 \mathrm{pa}-$ tients affected by type II hyperlipidemia (29 female and 21 male, mean age $58 \pm 8.2$ years) who did not respond to a 3-month low-fat diet, and 20 healthy volunteers ( 9 female and 11 male, mean age 56 \pm 11.2 years). Patients were eligible for the study if they met the following criteria: total plasma cholesterol level (TC) $\geq 250 \mathrm{mg} / \mathrm{dl}$, LDL-C $\geq 170 \mathrm{mg} / \mathrm{dl}$ and triglycerides (TG) $\leq 400 \mathrm{mg} / \mathrm{dl}$. The exclusion criteria were as follows: secondary dyslipidemia in the course of autoimmune disorders, thyroid diseases, chronic pancreatitis, nephritic syndrome, liver and biliary tract disease or alcoholism, any acute or chronic inflammatory disorders, congestive heart failure, coronary artery disease, history of myocardial infarction, stroke or intermittent claudication, arterial hypertension, diabetes mellitus, impaired renal or hepatic function, malignancy or history of malignancy, treatment with hypolipemic, hypotensive, anticoagulant, antiplatelet or fibrinolytic drugs, concomitant treatment with drugs that may affect inflammatory processes in the vascular wall (including non-steroidal anti-inflammatory drugs, angiotensin-converting enzyme inhibitors, antioxidant therapy, ongoing hormonal replacement therapy or oral contraception), abuse of alcohol, and poor patient compliance.

\section{Ethics}

All subjects provided written informed consent prior to study participation. The study was approved by the Local Bioethics Commission of the Lodz Medical University (approval no. WAM/1102).

\section{Study design}

Patients with hyperlipidemia $(n=50)$ were treated with statins: $10 \mathrm{mg}$ of atorvastatin $(n=20)$, $20 \mathrm{mg}$ of simvastatin $(n=18)$ or $20 \mathrm{mg}$ of pravastatin $(n=12)$ given once daily at bedtime for 8 weeks. Two control visits were scheduled for subjects: one before treatment initiation, and one after 8 weeks of therapy. During control visits, clinical examination and venous blood sampling for the evaluation of lipid profile, fibrinogen, and platelet adhesion and aggregation were performed, as well as diagnostic laboratory safety tests (aminotransferases, creatine kinase). The samples were immediately coded so that the laboratory assay was blinded to subject identity and study sequence. Compliance was assessed during each visit by tablet counts and was considered satisfactory when the number of tablets taken by a patient ranged from $90 \%$ to $100 \%$.

\section{Blood collection and preparation of platelets}

Blood samples were taken after an overnight fast in a quiet, temperature-controlled room $\left(24-25^{\circ} \mathrm{C}\right)$ between 8.00 and 9.00 a.m. (to avoid circadian fluctuations of fibrinogen). The blood was then stored in ACD (15 g/l citric acid, 20 g/l glucose, 25 g/l sodi- 
um citrate, $1: 5 \mathrm{v} / \mathrm{v}$ ) for platelet adhesion studies, in sodium citrate $(3.2 \%, 1: 9 \mathrm{v} / \mathrm{v})$ for aggregation and fibrinogen, and in serum vacutainers for lipid profile. Platelet rich plasma (PRP) was obtained by centrifugation $(150 \times \mathrm{g}, 10 \mathrm{~min}$, room temperature (RT)) within 60 min after blood collection. Platelet poor plasma (PPP) was obtained by subsequent centrifugation of PRP $(2500 \times \mathrm{g}, 20 \mathrm{~min})$. To preserve the platelet discoid shape, the PRP was restored in a water bath, during a resting period of $30 \mathrm{~min}$ at $37^{\circ} \mathrm{C}[10]$. The platelet count in the PRP was estimated routinely by photometry, using plastic cuvettes placed in a Cecil CE 1100 spectrophotometer (CECIL, Cambridge, UK), with a $10 \mathrm{~mm}$ optical path. The measurements were performed at $\lambda=800 \mathrm{~nm}[11]$. The PRP was subsequently diluted with the PPP to a concentration of approximately 250000 platelets $/ \mathrm{ml}$. The blood collected in ACD was also centrifuged $(150 \times \mathrm{g}, 10 \mathrm{~min}, \mathrm{RT})$, followed by centrifugation of the PRP $(700 \times \mathrm{g}, 15 \mathrm{~min}, \mathrm{RT})$, and the platelet pellet was resuspended in buffer A (pH 7.4; $145 \mathrm{mmol} / \mathrm{l} \mathrm{NaCl} ; 5 \mathrm{mmol} / \mathrm{l} \mathrm{KCl} ; 10 \mathrm{mmol} / \mathrm{l}$ HEPES; $0.5 \mathrm{mmol} / / \mathrm{Na}_{2} \mathrm{HPO}_{4} ; 6 \mathrm{mmol} / /$ glucose; $0.2 \%$ bovine serum albumin).

\section{Platelet aggregation assay}

The aggregation of platelets in the PRP was measured with Born's turbidimetric method [12], using the previously described aggregometer [13]. Aggregation curves were recorded and evaluated using our own computer software [14]. The instrument was set for $100 \%$ transmission (\%T) with PPP prior to each reading. To discourage the spontaneous aggregation of samples, the PRP was preincubated at $37^{\circ} \mathrm{C}$ for $3 \mathrm{~min}$, with stirring. The aggregation process was triggered by the addition of $5 \mu \mathrm{l}$ of a platelet agonist: ADP $(10 \mu \mathrm{mol} / \mathrm{l})$, collagen $(4 \mu \mathrm{g} / \mathrm{ml})$ or ristocetin $(2 \mathrm{mg} / \mathrm{ml})$ to the $495 \mu \mathrm{l}$ of PRP (in disposable plastic cuvettes with stirring at $800 \mathrm{rpm}$ ), and observed for a minimum of $5 \mathrm{~min}$ after reaching maximal aggregation. Our software estimated 5 parameters of platelet aggregation: maximal aggregation $\left(A_{\max }\right)$, initial velocity $\left(v_{0}\right)$, the time needed to reach maximal aggregation (Tmax), the aggregation level 5 min after Amax ( $A_{5 \min }$; to estimate disaggregation), and platelet shape change (PSC; only a rough estimation of this process) [14].

\section{Platelet adhesion}

Platelet adhesion to human fibrinogen coated in multiwell microplates was investigated using a microplate reader (Elx 800; Bio-Tek Instruments Inc., Winosski, Vermont, USA) at $405 \mathrm{~nm}$ as previously described [15]. In some cases, $10 \mu \mathrm{l}$ of ADP $(1 \mathrm{mmol} / \mathrm{l})$ was added to stimulate adhesion. The number of adherent platelets was estimated by the measurement of acid phosphatase activity - an unreleased platelet enzyme with stable activity independent of their stimulation [16].

\section{Other laboratory assays}

The other laboratory parameters were determined by routinely used methods at the Dr. W. Bieganski Voivodship Specialist Hospital in Lodz. The lipid profile, i.e. TC, TG, high-density lipoprotein cholesterol (HDL-C) and LDL-C levels, was determined by enzymatic methods (Olympus AU 400 analyzer; reagent kits from bioMerieux). Fibrinogen levels were measured according to the Clauss [17] method with the use of reagents supplied by Fibriquik ${ }^{\mathrm{TM}}$ and the Coag-A-Mate MTX analyzer (bioMerieux).

\section{Statistical analysis}

All values are expressed as a mean \pm SD. Statistical tests were performed using the Statistica 8.0 software package. All data were analyzed by ANOVA. Correlations between the change in lipid and fibrinogen levels, and the platelet activity parameters and statin treatment were determined by Pearson's correlation analysis. Values of $p \leq 0.05$ was considered significant.

\section{Results}

Main baseline characteristics of the included patients and the effects of statins on plasma lipid levels are shown in Table I. No significant adverse effects were recorded, and all patients completed the study. All laboratory safety measurements remained within normal limits.

Initial serum levels of TC, LDL-C, TG and the LDL-C/ $\mathrm{HDL}-\mathrm{C}$ ratio were significantly higher in all 3 groups of patients with hyperlipidemia compared with the control group ( $p<0.001)$. However, differences between groups of hyperlipidemic subjects were not observed. Serum HDL-C concentrations were comparable in patients with hyperlipidemia and in healthy controls. We did not notice any changes in serum HDL-C levels after therapy with statins. Upon the completion of 8-week atorvastatin, simvastatin and pravastatin administration, a significant and comparable decrease in TC, LDL-C, TG and the LDL-C/HDL-C ratio was observed.

Significant changes in some kinetic parameters of ADP-induced platelet aggregation after shortterm statin therapy were also observed. Mean values of the evaluated parameters of platelet aggregation at baseline and after 8 weeks of treatment by the three statins are presented in Table II. We did not observe any significant effect of statin therapy on ristocetin and collagen-induced platelet aggregation.

In patients with hyperlipidemia, significantly higher levels of fibrinogen before and after statin 
Table I. Lipid levels (mean \pm SD) at baseline and after 8 weeks treatment with atorvastatin (10 mg/day), simvastatin (20 $\mathrm{mg} /$ day) and pravastatin ( $20 \mathrm{mg} /$ day)

\begin{tabular}{|c|c|c|c|c|c|c|c|}
\hline \multirow{2}{*}{$\begin{array}{l}\text { Lipid } \\
\text { parameters }\end{array}$} & \multicolumn{2}{|c|}{ Atorvastatin $(n=20)$} & \multicolumn{2}{|c|}{ Simvastatin $(n=18)$} & \multicolumn{2}{|c|}{ Pravastatin $(n=12)$} & \multirow{2}{*}{$\begin{array}{l}\text { Control } \\
(n=20)\end{array}$} \\
\hline & Baseline & $\begin{array}{l}\text { 8-Week } \\
\text { treatment }\end{array}$ & Baseline & $\begin{array}{l}\text { 8-Week } \\
\text { treatment }\end{array}$ & Baseline & $\begin{array}{l}\text { 8-Week } \\
\text { treatment }\end{array}$ & \\
\hline $\begin{array}{l}\text { Total cholestero } \\
{[\mathrm{mg} / \mathrm{dl}]}\end{array}$ & $294 \pm 41.8^{+++}$ & $202 \pm 43.8^{\star \star \star /++}$ & $304 \pm 44.4^{+++}$ & $208 \pm 32.7^{* * * /++}$ & $296 \pm 31.1^{+++}$ & $224 \pm 35.7^{\star * \star /+++}$ & $188 \pm 16.4$ \\
\hline LDL-C [mg/dl] & $207 \pm 37.6^{+++}$ & $123 \pm 36.9^{* \star \star}$ & $211 \pm 29.8^{+++}$ & $126 \pm 28.0^{\star \star \star \star}$ & $206 \pm 29.8^{+++}$ & $139 \pm 38.5^{\star \star \star /+}$ & $117 \pm 12.9$ \\
\hline $\mathrm{HDL}-\mathrm{C}[\mathrm{mg} / \mathrm{dl}]$ & $54 \pm 15.1$ & $50 \pm 13.8$ & $57 \pm 10.6$ & $54 \pm 9.1$ & $54 \pm 10.43$ & $51 \pm 9.0$ & $50 \pm 11.2$ \\
\hline LDL/HDL ratio & $4 \pm 0.9^{+++}$ & $2 \pm 0.7^{\star \star \star}$ & $3 \pm 0.9^{+++}$ & $2 \pm 0.7^{\star \star \star \star}$ & $3 \pm 0.8^{+++}$ & $2 \pm 0.9^{\star \star \star}$ & $2 \pm 0.6$ \\
\hline TG [mg/dl] & $180 \pm 97.0^{+++}$ & $140 \pm 82.1^{\star \star /+}$ & $177 \pm 77.6^{+++}$ & $138 \pm 53.2^{\star * /+}$ & $166 \pm 79.0^{+++}$ & $142 \pm 53.2^{* * /+}$ & $102 \pm 24.4$ \\
\hline
\end{tabular}

${ }^{* *} p<0.05$ vs. baseline, ${ }^{* * *} p<0.001$ vs. baseline, ${ }^{+} p=0.05$ vs. control, ${ }^{++} p<0.05$ vs. control, ${ }^{+++} p<0.001$ vs. control. LDL-C - low-density lipoprotein cholesterol, HDL-C - high-density lipoprotein cholesterol, TG - triglycerides

Table II. Parameters of platelet aggregation (mean $\pm S D$ ): maximal aggregation $A_{\max }\left(\% T\right.$ ) and initial velocity $v_{0}$ (\%T/min)

\begin{tabular}{|lcccccccc|}
\hline $\begin{array}{l}\text { Aggregation } \\
\text { inductor }\end{array}$ & Parameter & \multicolumn{2}{c}{ Atorvastatin $(n=20)$} & Simvastatin $(n=18)$ & Pravastatin $(n=12)$ & $\begin{array}{c}\text { Control } \\
(n=20)\end{array}$ \\
\cline { 2 - 9 } & & Baseline & $\begin{array}{c}\text { 8-Week } \\
\text { treatment }\end{array}$ & Baseline & $\begin{array}{c}\text { 8-Week } \\
\text { treatment }\end{array}$ & Baseline & $\begin{array}{c}\text { 8-Week } \\
\text { treatment }\end{array}$ & \\
\hline ADP & $\mathrm{A}_{\max }[\% \mathrm{~T}]$ & $50.6 \pm 12.8$ & $41.1 \pm 15.8^{* *}$ & $57.2 \pm 18.0$ & $44.7 \pm 22.1^{*}$ & $55.8 \pm 19.5$ & $38.81 \pm 23.39^{\star * /+}$ & $48.6 \pm 15.4$ \\
& $v_{0}[\% \mathrm{~T} / \mathrm{min}]$ & $40.8 \pm 15.0$ & $32.9 \pm 14.7$ & $40.26 \pm 12.3$ & $32.4 \pm 18.9$ & $33.4 \pm 19.2$ & $24.92 \pm 13.35^{\star /++}$ & $37.2 \pm 14.7$ \\
\hline Ristocetin & $\mathrm{A}_{\max }[\% \mathrm{~T}]$ & $57.2 \pm 10.7$ & $54.8 \pm 20.3$ & $61.2 \pm 24.8$ & $60.1 \pm 20.9$ & $65.73 \pm 27.1$ & $58.83 \pm 26.0$ & $62.1 \pm 7.8$ \\
& $v_{0}[\% \mathrm{~T} / \mathrm{min}]$ & $39.4 \pm 13.4$ & $38.9 \pm 18.5$ & $35.3 \pm 9.8$ & $39.3 \pm 18.3$ & $37.9 \pm 18.4$ & $34.2 \pm 17.0$ & $41.2 \pm 13.6$ \\
\hline Collagen & $\mathrm{A}_{\max }[\% \mathrm{~T}]$ & $41.5 \pm 21.2$ & $43.0 \pm 20.8$ & $41.9 \pm 20.7$ & $39.8 \pm 23.5$ & - & - & $40.7 \pm 25.0$ \\
& $v_{O}[\% \mathrm{~T} / \mathrm{min}]$ & $29.7 \pm 17.5$ & $33.3 \pm 19.2$ & $27.1 \pm 17.5$ & $21.95 \pm 17.9$ & - & - & $27.0 \pm 18.2$ \\
\hline
\end{tabular}

${ }^{*} p=0.05$ vs. baseline, ${ }^{* *} p<0.05$ vs. baseline, ${ }^{+} p=0.05$ vs. control, ${ }^{++} p<0.05$ vs. control

Table III. Fibrinogen concentration and platelet adhesion to fibrinogen (mean \pm SD)

\begin{tabular}{|c|c|c|c|c|c|c|c|}
\hline \multirow[t]{2}{*}{ Parameter } & \multicolumn{2}{|c|}{ Atorvastatin $(n=20)$} & \multicolumn{2}{|c|}{ Simvastatin $(n=18)$} & \multicolumn{2}{|c|}{ Pravastatin $(n=12)$} & \multirow{2}{*}{$\begin{array}{l}\text { Control } \\
(n=20)\end{array}$} \\
\hline & Baseline & $\begin{array}{l}\text { 8-Week } \\
\text { treatment }\end{array}$ & Baseline & $\begin{array}{l}\text { 8-Week } \\
\text { treatment }\end{array}$ & Baseline & $\begin{array}{l}\text { 8-Week } \\
\text { treatment }\end{array}$ & \\
\hline Fibrinogen $[\mathrm{g} / \mathrm{l}]$ & $362 \pm 70.1^{+}$ & $380 \pm 88.3^{+}$ & $381 \pm 72.2^{++}$ & $340 \pm 77.7^{+}$ & $379 \pm 73.2^{+}$ & $358 \pm 82.9^{+}$ & $318 \pm 62.8$ \\
\hline $\begin{array}{l}\text { Spontaneous } \\
\text { adhesion [\%] }\end{array}$ & $8.2 \pm 5.25$ & $10.9 \pm 5.96$ & $8.1 \pm 8.0$ & $10.2 \pm 5.0$ & $8.7 \pm 6.7$ & $7.5 \pm 4.0$ & $9.37 \pm 6.95$ \\
\hline $\begin{array}{l}\text { ADP-induced } \\
\text { adhesion [\%] }\end{array}$ & $16.1 \pm 6.48$ & $19.0 \pm 5.89$ & $17.1 \pm 11.9$ & $17.7 \pm 4.2$ & $15.9 \pm 6.4$ & $17.41 \pm 7.7$ & $17.51 \pm 5.06$ \\
\hline
\end{tabular}

${ }^{+} p=0.05$ vs. control, ${ }^{++} p<0.05$ vs. control

therapy were observed when compared to the control group. However, both spontaneous and induced adhesions of platelets to fibrinogen were comparable in each group; they did not change after the 8 weeks of statin therapy (Table III). However, at baseline, positive correlations were found between the ADP-induced adhesion to fibrinogen and the plasma concentration of TC $(r=0.512, p=0.007)$, together with LDL-C $(r=0.428, p=0.006)$. At the end of 8 weeks of statin treatment, no significant correlations between lipid parameters and platelet adhesion were found. At baseline, no significant correlations were observed between fibrinogen concentration and spontaneous adhesion to fibrinogen $(r=-0.039, p=0.860)$, or with ADP-induced adhesion to fibrinogen $(r=0.052, p=0.817)$.

\section{Discussion}

In the last decades, the beneficial effects of statins (3-hydroxy-methylglutaryl coenzyme A reductase inhibitors) in the prophylaxis and treatment of cardiovascular disease have been corroborated in many large controlled studies [18-21].

The list of effects of statins, other than hypolipemic, is still expanding and includes: a decrease in expression or activity of inflammatory agents and metalloproteinases, and an influence on endothelial function and the hemostatic system [22]. Results of studies on the effects of statin therapy on platelet function depend on the dose of statin, method of evaluation of platelet activity, and the use of agonists and other agents (e.g. additional 
antiplatelet therapy) [23]. Platelet hyperactivity after discontinuance of statin therapy was reported [24]. This may contribute to the increased risk of events that may occur after the discontinuation of statin treatment in high risk patients [25]. There were suggested different mechanisms of statin influence on platelet activity. They could be either dependent or independent of the cholesterol level changes. Decreased platelet stimulation by LDL and their oxidation products, lowering of cholesterol concentration in the platelet plasma membrane, attenuation of signal transduction due to a decrease in intracellular isoprenoid and $A_{2}$ thromboxane production and increased NO bioavailability may all be responsible for the decreased platelet activation [23-26].

In our studies we reported that even short-term therapy with a low dose of atorvastatin, simvastatin or pravastatin favorably influenced plasma lipid levels in patients with newly diagnosed hyperlipidemia. Significant reductions in TC levels $(p<0.001), \mathrm{LDL}-\mathrm{C}(p<0.001)$, the LDL-C/HDL-C ratio $(p<0.001)$ and even TG were observed.

Moreover, our data confirm previous observations that simvastatin and pravastatin have little or no fibrinogen-lowering effect, whereas atorvastatin induced a non-significant increase in fibrinogen, thus confirming its previously described time and dose-related effects [27]. Bolaman et al. [28] reported a $23 \%$ increase in fibrinogen levels after 24 weeks of atorvastatin intake at a dose of $10 \mathrm{mg}$ a day. The increase was more significant in females. Our results also confirm this observation, since women constituted $65 \%$ of the studied group and the increase in fibrinogen levels was greater in this group. However, some authors suggest that the effect of atorvastatin on plasma fibrinogen levels in dyslipidemic patients without evident vascular disease is not clinically relevant. Furthermore, any rise in fibrinogen levels that may occur is likely to be transient in nature [29].

Spontaneous platelet adhesion to fibrinogen, which may indirectly indicate in vivo conditions, and ADP-induced adhesion determining platelet activity in vitro, were evaluated in our study. No relevant influence of the 8-week atorvastatin, simvastatin or pravastatin therapy on the spontaneous or ADPactivated adhesion of platelets to fibrinogen was observed. However, a strong positive correlation between lipids (TC, LDL-C) and the extent of ADPactivated adhesion in patients with hyperlipidemia before the introduction of the statin therapy was reported. We found no correlation between adhesion and the lipid concentrations after statin therapy. It is possible that the increase in the fibrinogen levels is responsible for the lack of changes in the extent of adhesion, in spite of the significant reduction of lipid concentration. It is essential because an undesirable increase in adhesion is a predictor of atherosclerosis [15, 30].

In our experiments we applied commonly used stimulants: ADP, collagen and ristocetin. The aggregation curve depends on the stimulant and concentration used. Generally, at $10 \mu \mathrm{mol} / \mathrm{L}$ ADP or $4 \mu \mathrm{g} /$ $\mathrm{ml}$ collagen concentrations, platelets release granules and form aggregates that do not dissociate (secondary aggregation) [31].

In our study we observed a slight initial enhancement of ADP-induced maximal platelet aggregation in hyperlipidemic patients compared with healthy controls. The decrease of maximal aggregation (Amax) and slowing down of this process ( $v_{0}$ decrease) suggest that statin therapy significantly inhibits platelet aggregation. Moreover, significantly lower Amax values in hyperlipidemic patients after pravastatin administration in comparison with the control group indicates that this particular statin has the strongest effect. After therapy with pravastatin a significant $25 \%(p=0.05)$ slowing down of platelet aggregation $\left(v_{0}\right)$ was observed. However, there were no differences in the kinetic parameters of collagen-induced platelet aggregation between healthy controls and patients with hyperlipidemia. Similarly, therapy with statins did not affect it either.

There are many general reviews [8,9], but only a few actual reports describe the effect of statins on platelet function [22]. The influence of statins on aggregation was evaluated by other investigators with the use of different agonists and methods, but the results are conflicting. Piotrowski et al. [23] compared the effects observed in patients with coronary artery disease after $40 \mathrm{mg} /$ day atorvastatin or $10 \mathrm{mg} /$ day of atorvastatin with ezetimibe. In both groups comparable hypolipemic effects were obtained. Additionally, maximal ATP or TRAP (thrombin receptor activating peptide)-induced aggregation was assessed. In the group of patients using $10 \mathrm{mg} /$ day of atorvastatin with ezetimibe no influence on the maximal aggregation was observed. In the group using higher doses of the statin only, TRAP-induced aggregation was reduced significantly. Therefore, the authors suggested that the effects of high doses of statins depend on their extra-lipid activity, which is not noticed at lower doses. On the other hand, Hussain et al. [32] showed that treatment with ezetimibe $(10 \mathrm{mg})$ in hypercholesterolemia was associated with decreased platelet aggregation and LDL tendency to peroxidation. Moreover, treatment with ezetimibe in addition to simvastatin $(20 \mathrm{mg}$ ) has an additive antioxidative effect on LDL.

Dujovne et al. [33], through the evaluation of the effects of atorvastatin therapy at a dose of $80 \mathrm{mg} /$ day for 12 weeks in patients with primary hyperlipoproteinemia, investigated aggregation in the whole blood with the impedance method, using 
ADP, collagen and arachidonic acid (AA). Despite the use of high doses of atorvastatin, no inhibiting effects on aggregation were observed in the case of ADP and collagen use. Only AA-induced aggregation significantly decreased by $11 \%$. In another report, a significant reduction in ADP-induced aggregation in patients with coronary artery disease was observed after 2 months of atorvastatin treatment. No changes in collagen or epinephrine-induced aggregation were found [27].

There are no studies evaluating the influence of statin therapy on ristocetin-induced aggregation. In vitro ristocetin-induced platelet aggregation involves platelet agglutination - caused directly by von Willebrand factor (VWF) binding to glycoprotein GPIb-IX. In vivo, under high shear rate flow conditions, interaction between subendothelial-bound VWF and its receptor caused platelet adhesion to the subendothelium. Besides its function in platelet aggregation, it may also play a role in the pathogenesis of atherosclerosis. Increased levels of vWF may indicate endothelial damage with a risk of atherosclerosis $[34,35]$. In the group of studied subjects, no relevant influence of statin therapy on the kinetic parameters of ristocetin-induced aggregation was observed. No correlation between these parameters and lipid concentration or fibrinogen levels, both before and after treatment, was found. It suggests that there was no influence on the ristocetin-induced platelet agglutination, and vWF levels probably did not change after the atorvastatin treatment.

The considerable differences in the extra-lipid effects of various statins, described in many studies, may be caused by different factors. It should be noted that studied subjects often suffer from concomitant diseases and take other medications, including antiplatelet drugs. Moreover, differences may also result from the different methods of platelet activity assessment.

Only individuals with newly diagnosed hyperlipidemia, without concomitant diseases and who did not take any additional medications took part in our study. Atherosclerotic processes are likely to be less advanced in this population. Furthermore, the increase in fibrinogen levels might, to some extent, have influenced the poor effectiveness of statins with regard to changes in platelet activity.

We are aware that our study has some limitations. The small number of subjects and the design of the study (a placebo-treated group was not included) are both limiting factors.

In conclusion, therapy with statins, regardless of the statin type, beneficially modifies ADP-induced platelet aggregation in patients with hyperlipidemia and no concomitant diseases. However, such treatment did not affect spontaneous or ADP-induced platelet adhesion to fibrinogen, or platelet aggregation induced by collagen or ristocetin.

\section{References}

1. Kamath S, Blann AD, Lip GYH. Platelet activation: assessment and quantification. Eur Heart J 2001; 22: 1561-71.

2. Hartwich J, Dembińska-Kieć, Gruca A, et al. Regulation of platelet adhesion by oxidized lipoproteins and oxidized phospholipids. Platelets 2002; 13: 141-51.

3. Cha YJ, Chang EA, Kim C. Effects of endothelial cells and mononuclear leukocytes on platelet aggregation. Haematologia 2000; 30: 97-106.

4. Gaddam V, Li DY, Metha JL. Anti-thrombotic effects of atorvastatin-an effect unrelated to lipid lowering. J Cardiovasc Pharmacol Therapeut 2002; 7: 247-53.

5. van Willigen G, Gorter G, Akkerman JW. LDLs increase the exposure of fibrinogen binding sites on platelets and secretion of dense granules. Arterioscler Thromb 1994; 14: 41-6.

6. Hackeng CM, Huigsloot M, Pladet MW, Nieuwenhuis HK, van Rijn HJM, Akkerman JWN. Low-density lipoprotein enhances platelet secretion via integrin-alphallbbeta3mediated signaling. Arterioscler Thromb Vasc Biol 1999; 19: 239-47.

7. Vlasova II. The effect of oxidatively modified low-density lipoproteins on platelet aggregability and membrane fluidity. Platelets 2000; 11: 406-14

8. Chalubinski M, Broncel M. The influence of statins on effector and regulatory immune mechanisms and their potential clinical relevance in the treatment of autoimmune disorders. Med Sci Monit 2010; 16: 11.

9. Takemoto M, Liao JK. Pleiotropic effects of 3-hydroxy3-methylglutaryl coenzyme A reductase inhibitors. Atheroscler Thromb Vasc Biol 2001; 21: 1712-9.

10. Maurer-Spurej E, Pfeiler G, Maurer N, Lindner H, Glatter O, Devine DV. Room temperature activates human blood platelets. Lab Invest 2001; 8: 581-92.

11. Walkowiak B, Michalak E, Koziołkiewicz W, Cierniewski CS. Rapid photometric method for estimation of platelet count in blood plasma in platelet suspension. Thromb Res 1989; 56: 763-6.

12. Born GV. Aggregation of blood platelets by adenosine and reversal. Nature 1962; 195: 927-9.

13. Kostka B, Pająk W. The aggregometer equipment for recording of transmittance $(T)$ with the possibility of scale extension. Diagn Lab 1985; 21: 43-7.

14. Kostka B, Sikora J, Para J, Krajewska U, Korzycka L. A new nitrate derivative of piperazine: its influence on platelet activity. Blood Coagul Fibrinol 2007; 18: 151-6.

15. Sikora J, Krajewska U, Gietka M, Kostka B. The application of pig blood in the in vitro measurement of platelet adhesion. Pharmazie 2001; 56: 187-8.

16. Bellavite P, Andrioli G, Guzzo P, et al. A colorimetric method for the measurement of platelet adhesion in plates. Anal Biochem 1994; 216: 444-50.

17. Clauss A. Rapid physiological coagulation method for the determination of fibrinogen. Acta Haemat 1957; 17: 237.

18. Balk EM, Lau J, Gaudas LC, et al. Effects of statins on nonlipid serum markers associated with cardiovascular disease. Ann Intern Med 2003; 139: 670-82.

19. Veselka J, Hájek P, Malý, et al. Predictors of coronary intervention-related myocardial infarction in stable angina patients pre-treated with statins. Arch Med Sci 2011; 7 : 67-72.

20. Xu M, Yuan G, Wei F. Effect of atorvastatin in patients with chronic heart failure - insights from randomized clinical trials Arch Med Sci 2010; 6: 866-73.

21. Athyros VG, Tziomalos K, Gossios TD, et al.; GREACE Study Collaborative Group. Safety and efficacy of long-term 
statin treatment for cardiovascular events in patients with coronary heart disease and abnormal liver tests in the Greek Atorvastatin and Coronary Heart Disease Evaluation (GREACE) Study: a post-hoc analysis. Lancet 2010; 376: 1916-22.

22. Puccetti L, Pasqui AL, Auteri A, Bruni F. Mechanisms for antiplatelet action of statins. Curr Drug Targets Cardiovasc Haematol Disord 2005; 5: 121-6.

23. Piotrowski M, Fisher S, Stellbaum C, et al. Treatment with ezetimibe plus low-dose Atorvastatin compared with higher-dose Atorvastatin alone. J Am Coll Cardiol 2007; 49: 1035-42.

24. Puccetti L, Pasqui AL, Pastorelli M, et al. Platelet hyperactivity after statin treatment discontinuation. Thromb Haemost 2003; 90: 476-82.

25. Tziomalos K, Athyros VG, Mikhailidis DP. Statin discontinuation: an underestimated risk? Curr Med Res Opin 2008; 24: 3059-62.

26. Jagroop IA, Persaud JW, Mikhailidis DP. A new rapid method to measure human platelet cholesterol: a pilot dtudy. Clin Appl Thromb Hemost 2011; 17: 578-84.

27. Puccetti L, Pasqui AL, Pastorelli M, et al. Time-dependent effect of statins on platelet function in hypercholesterolaemia. Eur J Clin Invest 2002; 32: 901-8.

28. Bolaman Z, Kadikoylu G, Ozgel N, Yenisey C. Effects of atorvastatin on coagulation parameters and homocysteine in patients with primary hypercholesterolemia. J Natl Med Assoc 2006; 98: 1273-7.

29. Goudevenos JA, Bairaktari ET, Chatzidimou KG, Milionis HJ, Mikhailidis DP, Elisaf MS. The effect of atorvastatin on serum lipids, lipoprotein(a) and plasma fibrinogen levels in primary dyslipidaemia: a pilot study involving serial sampling. Curr Med Res Opin 2001; 16: 269-75.

30. Bonnefoy A, Liu Q, Legrand, Frojmovic MM. Efficiency of platelet adhesion to fibrinogen depends on both cell activation and flow. Biophysical J 2000; 78: 2834-43.

31. Ozaki Y. Measurement of platelet aggregation and attempts for standardization. Sysmex J Inter 1998; 8: 15-22.

32. Hussain O, Minasian L, Itzkovich Y, Shestatski K, Solomon L, Zidan J. Ezetimibe's effect on platelet aggregation and LDL tendency to peroxidation in hypercholesterolaemia as monotherapy or in addition to simvastatin. $\mathrm{Br}$ J Clin Pharmacol 2008; 65: 637-45.

33. Dujove CA, Harris WS, Altman R, Overhiser RW, Black DM. Effect of atorvastatin on hemorheologic-hemostatic parameters and serum fibrinogen levels in hyperlipidemic patients. Am J Cardiol 2000; 85: 350-3.

34. Dai K, Bodnar R, Berndt MC, Du X. A critical role for 14-33zeta protein in regulating the VWF binding function of platelet glycoprotein Ib-IX and its therapeutic implications. Blood 2005; 106: 1975-81.

35. Szapary L, Horvayh B, Marton Z, et al. Short-term effects of low-dose Atorvastatin on haemorrheological parameters, platelet aggregation and endothelial function in patients with cerebrovascular disease and hyperlipidaemia. CNS Drugs 2004; 18: 165-72. 